\title{
The Keck I/HIRES and TNG/SARG Radial Velocity Survey of Speckle Binaries
}

\author{
Milena Ratajczak ${ }^{1}$, Maciej Konacki ${ }^{1,2}$, Shrinivas R. Kulkarni ${ }^{3}$ \\ and Matthew W. Muterspaugh ${ }^{4}$ \\ ${ }^{1}$ Nicolaus Copernicus Astronomical Center, Polish Academy of Sciences, \\ ul. Rabiańska 8, 87-100 Toruń, Poland, \\ email: milena@ncac.torun.pl \\ ${ }^{2}$ Astronomical Observatory of Adam Mickiewicz University, \\ ul. Sloneczna 36, 60-286 Poznań, Poland \\ ${ }^{3}$ California Institute of Technology, Division of Physics, Mathematics and Astronomy, \\ Pasadena, CA 91125, USA \\ ${ }^{4}$ Tennessee State University, Department of Mathematics and Physics, \\ College of Arts and Sciences, \\ Boswell Science Hall, Nashville, TN 37209, USA
}

\begin{abstract}
A sample of about 160 speckle binary stars was observed with the Keck I telescope and its Échelle HIRES spectrograph over the years 2003-2007 in an effort to detect substellar and planetary companions to components of binary and multiple star systems. This data set was supplemented with the data obtained at the TNG telescope equipped with the SARG Échelle spectrograph over the years 2006-2007. The high-resolution $(\mathrm{R}=65000$ for HIRES and $\mathrm{R}=$ 86000 for SARG) and high signal-to-noise (typically 75-150) spectra were used to derive radial velocities of the components of the observed speckle binaries. Here, we present a summary of this effort, which includes the discovery of new triple star systems and improved orbital solutions of a few known binaries.
\end{abstract}

Keywords. stars: binaries: visual, techniques: radial velocities

\section{Method}

Precise radial velocities (RV) of stars are commonly obtained using an iodine (I2) absorption cell (Marcy \& Butler 1992). This classic approach is well-suited to measure RVs of single stars only. We developed a novel variant employing an I2 absorption cell which allows us to measure RVs of both components of double-lined binaries (SB2; Konacki 2005 ) and used it on a sample of $\sim 160$ speckle binaries to detect new stellar and substellar companions to members of known binary systems. The classical approach with the iodine cell cannot be used in the case of binary stars because it is not possible to observationally obtain two separate template spectra of binary components. The procedure is as follows (Konacki 2005):

(a) two exposures, with and without the I2 cell, are always taken to obtain an instantaneous template used to model the immediate exposure taken with the cell;

(b) a least squares fit is carried out to determine the parameters: $\Delta \lambda_{s}$ (shift of the star spectrum), $\Delta \lambda_{I 2}$ (shift of the iodine transmission function), and PSF using the observed (with and without the I2 cell) spectra. An extracted stellar spectrum has an accurate wavelength solution and it is free of the I2 lines and the influence of a varying PSF;

(c) two-dimensional cross correlation TODCOR (Zucker \& Mazeh 1994) is used on the extracted spectrum to obtain RVs of the components. To this end, synthethic spectra 
computed with ATLAS9 and ATLAS12 (Kurucz 1995) are used. The formal errors of RVs are derived using the TODCOR formalism.

\section{Example results}

Below, we present the analysis of 2 speckle binary systems. HD88417 (Fig. 1) is a newly discovered triple system, HD185082 (Fig. 2) is a triple system for which we derived the tertiary's orbit for the first time. For many other observed systems, we were able to obtain improved orbits of the components (the rms of our orbital fits in a few cases is even 100 times lower compared with the literature data).

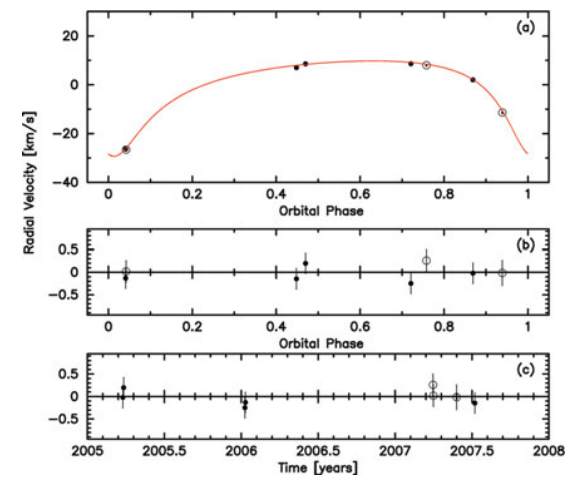

\section{HD88417}

WDS J10121+2118AB, HIP 49970 $(\mathrm{V}=8.8 \mathrm{mag})$, is a speckle binary with an orbital period of 97 years, semi-major axis of $0.2 \operatorname{arcsec}$ and an eccentricity of 0.26 (Heintz 1976; Hartkopf et al. 2001). Our measurements enable us to establish the binarity of the secondary and clasify it as a triple system.

Figure 1. RV $(a)$ and $\mathrm{rms}(b)$ : as a function of orbital phase, $(c)$ : as a function of time, of our orbital fit of the newly discovered component of HD88417.

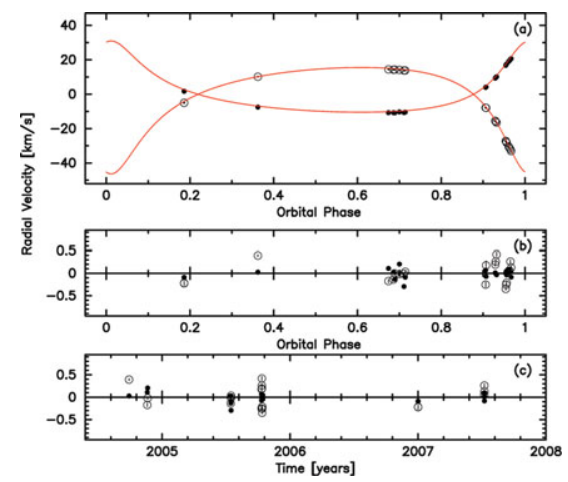

\section{HD185082}

WDS J19351+5038, HIP $96321(\mathrm{~V}=8.3$ $\mathrm{mag}$ ), is a speckle binary with an orbital period of 103 years, semi-major axis of 0.3 arcsec and an eccentricity of 0.58 (Hartkopf et al. 2001). The binarity of the primary was established by Smekhov 1999 who derived its spectroscopic orbit. Our RV measurements allow us to improve the orbital parameters significantly. In particular, the rms of the orbital fit for the primary is $0.1 \mathrm{~km} / \mathrm{s}$ compared to $1.3 \mathrm{~km} / \mathrm{s}$ of Smekhov 1999. Additionally, we were

Figure 2. RV ( $a$ ) and rms (b): as a function of orbital able to detect the lines of the tertiary phase, $(c)$ : as a function of time, of our orbital fit of (the spectroscopic companion of the both components of the primary of HD185082. primary) and derive its orbit as well.

\section{References}

Hartkopf, W. I., Mason, B. D., \& Worley, C. E. 2001a, 6th Catalog of Orbits of Visual Binary Stars

Heinz, W. D. 1976, ApJ, 208, 474

Konacki, M. 1995, ApJ, 626, 431

Kurucz R. L. 1995, ASP-CP, 78

Marcy, G. W. \& Butler R. P. 1992, PASP, 104, 270

Smekhov, M. G. 1999, Astron. Lett., 25, 536

Zucker, S. \& Mazeh, T. 1994, ApJ, 420, 806 